Article

\title{
Averaging Pixel Current Adjustment Technique for Reducing Fixed Pattern Noise in the Bolometer-Type Uncooled Infrared Image Sensor
}

\author{
Sang-Hwan Kim ${ }^{1}{ }^{10}$, Byoung-Soo Choi ${ }^{1}$, Jimin Lee ${ }^{1}$, Junwoo Lee ${ }^{1}$, Jewon Lee ${ }^{1}$, \\ Jae-Hyoun Park ${ }^{2}$, Kyoung-Il Lee ${ }^{2}$ and Jang-Kyoo Shin ${ }^{1, *(1)}$ \\ 1 School of Electronics Engineering, Kyungpook National University, Daegu 41566, Korea; \\ shkim7@knu.ac.kr (S.-H.K.); bschoi@ee.knu.ac.kr (B.-S.C.); jmLee@ee.knu.ac.kr (J.L.); \\ junwoolee@knu.ac.kr (J.L.); jewonlee@knu.ac.kr (J.L.) \\ 2 Korea Electronics Technology Institute, Seongnam-si 13509, Korea; jhpark@keti.re.kr (J.-H.P.); \\ leeki@keti.re.kr (K.-I.L.) \\ * Correspondence: jkshin@ee.knu.ac.kr; Tel.: +82-53-950-5531
}

Received: 28 February 2019; Accepted: 4 April 2019; Published: 6 April 2019

\begin{abstract}
In this paper, we propose an averaging pixel current adjustment technique for reducing fixed pattern noise (FPN) in the bolometer-type uncooled infrared image sensor. The averaging pixel current adjustment technique is composed of active pixel, reference pixel, and calibration circuit. Polysilicon resistors were used in each active pixel and reference pixel. Resistance deviation among active pixels integrated with the same resistance value cause FPN. The principle of the averaging pixel current adjustment technique for removing FPN is based on the subtraction of dark current of the active pixel from the dark current of the reference pixel. The subtracted current is converted into the voltage, which contains pixel calibration information. The calibration circuit is used to adjust the calibration current. After calibration, the nano-ampere current is output with small deviation. The proposed averaging pixel current adjustment technique is implemented by a chip composed of a pixel array, a calibration circuit, average current generators, and readout circuits. The chip was fabricated using a standard $0.35 \mu \mathrm{m}$ CMOS process and its performance was evaluated.
\end{abstract}

Keywords: uncooled infrared image sensor; fixed pattern noise; current calibration

\section{Introduction}

The bolometer-type uncooled infrared image sensor has recently been researched in various fields such as defense, medical care, and non-destructive testing [1-3]. Uncooled infrared image sensors have many advantages in terms of low power consumption, easy integration, and low cost [4-11]. Among the various uncooled infrared image sensors, the bolometer sensor based on micromachining technology has a higher temperature coefficient of resistance than other sensors. The response of a bolometer sensor, which is one type of thermal detector, is slow but it does not depend on the wavelength of infrared light and it can be used at room temperature. Therefore, there is no need for a thermoelectric cooler that consumes additional power and volume. In addition, various readout integrated circuits (ROICs) for bolometer-type uncooled infrared image sensors using a standard CMOS process have been developed [12-14]. Among the various ROICs, the capacitive transimpedance amplifier (CTIA) is widely used to detect the signal output current of pixels [15]. The bolometer sensor based on micromachining technology is compatible with the standard CMOS process, but low linearity and high noise are disadvantages.

Resistance deviation among the resistors integrated in the active pixel and reference pixel causes serious fixed pattern noise (FPN). In a readout circuit and a signal processing circuit integrated using 
a standard CMOS process, a method for compensating for these deviations is necessary in order to obtain an efficient infrared image. Various methods have been researched to solve the problem of the deviation between resistors integrated in the active pixel and reference pixel [16-18].

In this research, in order to compensate the resistance deviation occurring among the pixels of the bolometer-type uncooled infrared image sensor, the averaging pixel current adjustment technique was used, which reduces the dark current by averaging the currents generated in the bolometers of reference pixels in column readout circuits. For verifying the proposed averaging pixel current adjustment technique, the output nodes of each reference pixel are all tied. As a result, the output current of the reference pixel in which the light receiving portion is blocked to avoid being affected by the incident light is averaged. The bolometer resistor in the active pixels and reference pixels has a dark current component. When the current from each active pixel array is subtracted from the current from the reference pixel, the dark current of the active pixel can be removed. The averaging pixel current adjustment technique consists of a reference pixel, active pixel, calibration circuit, and readout circuit. The entire chip based on the averaging pixel current adjustment technique was fabricated using a $0.35 \mu \mathrm{m}$ standard CMOS process and its characteristics were analyzed.

\section{Principle of Pixel Averaging Pixel Current Adjustment Technique}

Figure 1a shows the schematic of the averaging pixel current adjustment technique. Each unit pixel is composed of an active pixel, reference pixel and calibration circuit. The bolometer resistors in the active pixel and reference pixel were integrated using resistors. Figure $1 \mathrm{~b}$ shows the modeling of the averaging pixel current adjustment technique. Kirchhoff's Current Law (KCL) is applied to Node A in Figure $1 \mathrm{a}$, and the output dark current of the active pixel $\left(I_{A C T}\right)$ can be subtracted from the output dark current of the reference pixel $\left(I_{R E F}\right)$. Additionally, the output current of the calibration circuit $\left(I_{C A L}\right)$ can be adjusted to account for reducing the dark current component. The current obtained by subtracting the dark current of the active pixel from the dark current of the reference pixel $\left(I_{R E F}-I_{A C T}\right)$ has deviation information that is generated by the resistance of the active pixel. The principle of detection and calibration for dark current is explained as follows:

$$
\begin{gathered}
I_{R E F} \propto \frac{1}{R_{R E F}} \\
I_{A C T} \propto \frac{1}{R_{A C T}} \\
I_{A C T}+\Delta I_{A C T}^{\prime} \propto \frac{1}{R_{A C T}+\Delta R_{A C T}^{\prime}} \\
I_{R E F}-I_{A C T}+\Delta I_{A C T}^{\prime}-I_{C A L} \triangleq \mathrm{nA} \text { order }
\end{gathered}
$$

where $\Delta R_{A C T}^{\prime}$ is the active bolometer resistance that is occurs by a process deviation and $\triangle I^{\prime}{ }_{A C T}$ is the deviation of the output dark current of the active pixel. In a unit pixel, the output dark current of the reference pixel $\left(I_{R E F}\right)$ is inversely proportional to the reference bolometer resistance $\left(R_{R E F}\right)$ and the output dark current of the active pixel $\left(I_{A C T}\right)$ is inversely proportional to the active bolometer resistance $\left(R_{A C T}\right)$. The $I_{A C T}$ changes when the $R_{A C T}$ in all the unit pixels is changed by the process deviation. Therefore, the $\triangle I^{\prime}{ }_{A C T}$ that causes the FPN is generated. Additionally, by reducing the $\triangle I^{\prime}{ }_{A C T}$ using the current of the calibration circuit $\left(I_{C A L}\right)$, the final output current can be reduced to nA order.

The deviation information is converted to voltage and digital codes through a current-voltage converter and an analog-digital converter (ADC), respectively. This deviation information causes the FPN. The $I_{C A L}$ proportional to the output code of the analog digital converter reduces the $I_{A C T}$. At this time, if $I_{A C T}$ is designed to always be larger or smaller than $I_{R E F}$, the direction of $I_{C A L}$ can be determined in one direction. When the output code of the ADC is controlled so that $I_{C A L}$ is almost equal to $I_{R E F}-I_{A C T}$, the pixel current offset due to the deviation of each active bolometer resistance is 
eliminated. Considering the characteristics of the current voltage converter, $I_{C A L}$ is controlled so that the output current offset of each active pixel is nA order.

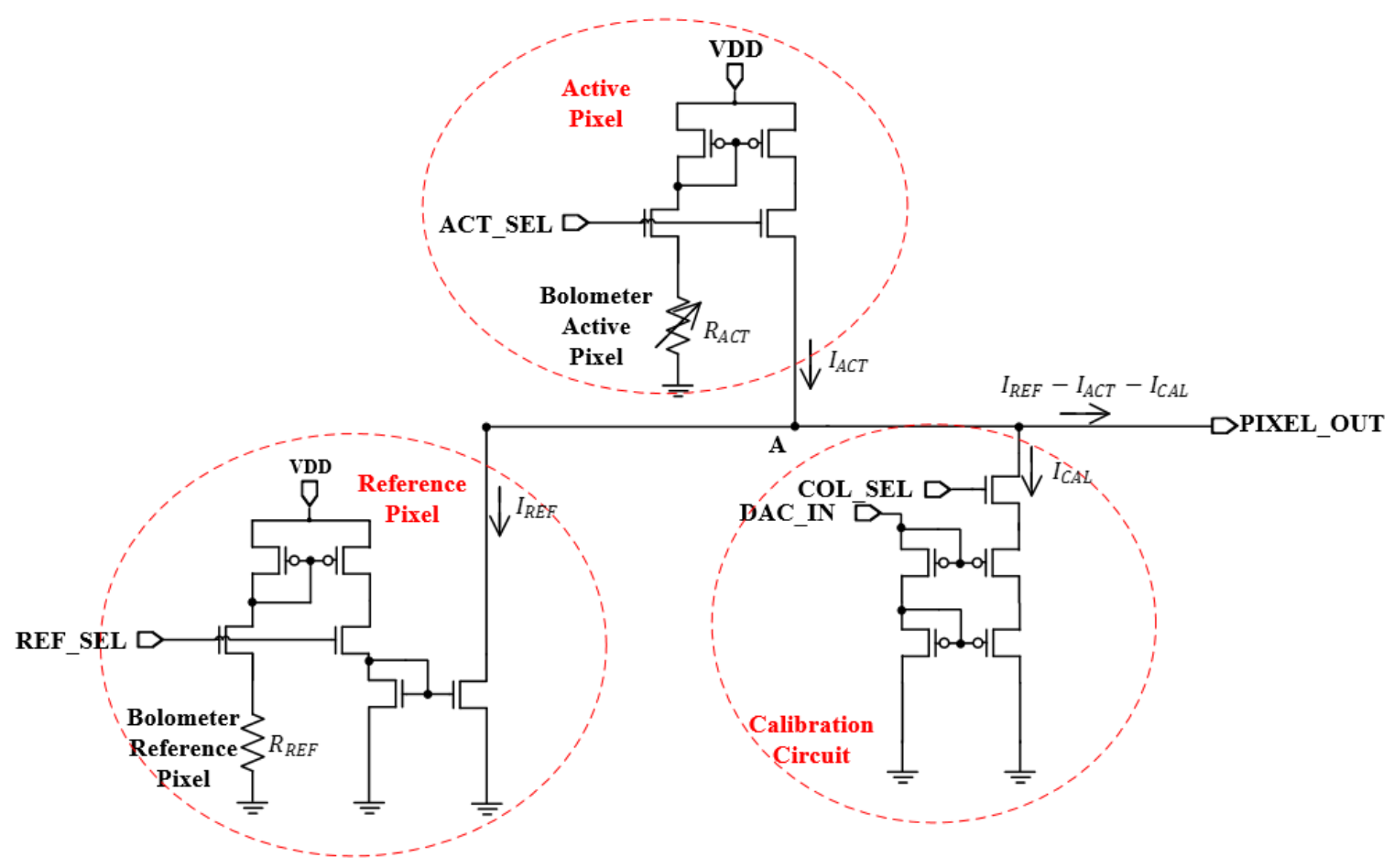

(a)

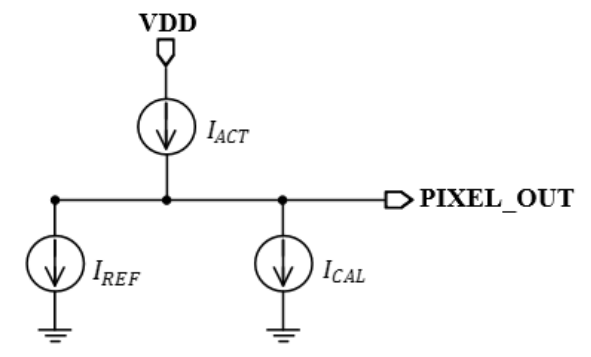

(b)

Figure 1. (a) Schematic of averaging pixel current adjustment technique (b) modeling of averaging pixel current adjustment technique.

In order to obtain the deviation information based on the resistance of the active pixel using the proposed averaging pixel current adjustment technique, the resistance of the reference pixel must be fixed without any deviation. However, not only the resistor of the active pixel has a process deviation, but also the resistor of the reference pixel has a process deviation. Figure 2a,b show a block diagram and a schematic diagram of the averaging current generator using the reference pixel, respectively. The average current generator was used to remove the offset current caused by resistance deviation among the resistors integrated in the active pixel. The principle for removing the offset current of the active pixel using the average current generator is as follows. First, output dark currents $\left(I_{R E F_{-} 0}, I_{R E F_{-} 1}\right.$, $\left.I_{R E F_{-} N}\right)$ are generated at Node $B$ of the reference pixels in each unit pixel. At this time, the output dark currents do not have uniform values due to the process deviation of the reference bolometer in each reference pixel. Second, output Node B of all the reference pixels is shared so that the output dark currents have an averaged value. Therefore, the average current $\left(\sum_{i=0}^{N} I_{R E F_{-} i} / N\right)$ at node $\mathrm{C}$ can be obtained. Finally, the average current is used as the output dark current of the reference pixel $\left(I_{R E F}\right)$ 
in Figure 1a. When the number of reference pixels increases, the average current converges to the average value. In this way, when the active pixel current $\left(I_{A C T}\right)$ is subtracted using the average current, FPN is removed.

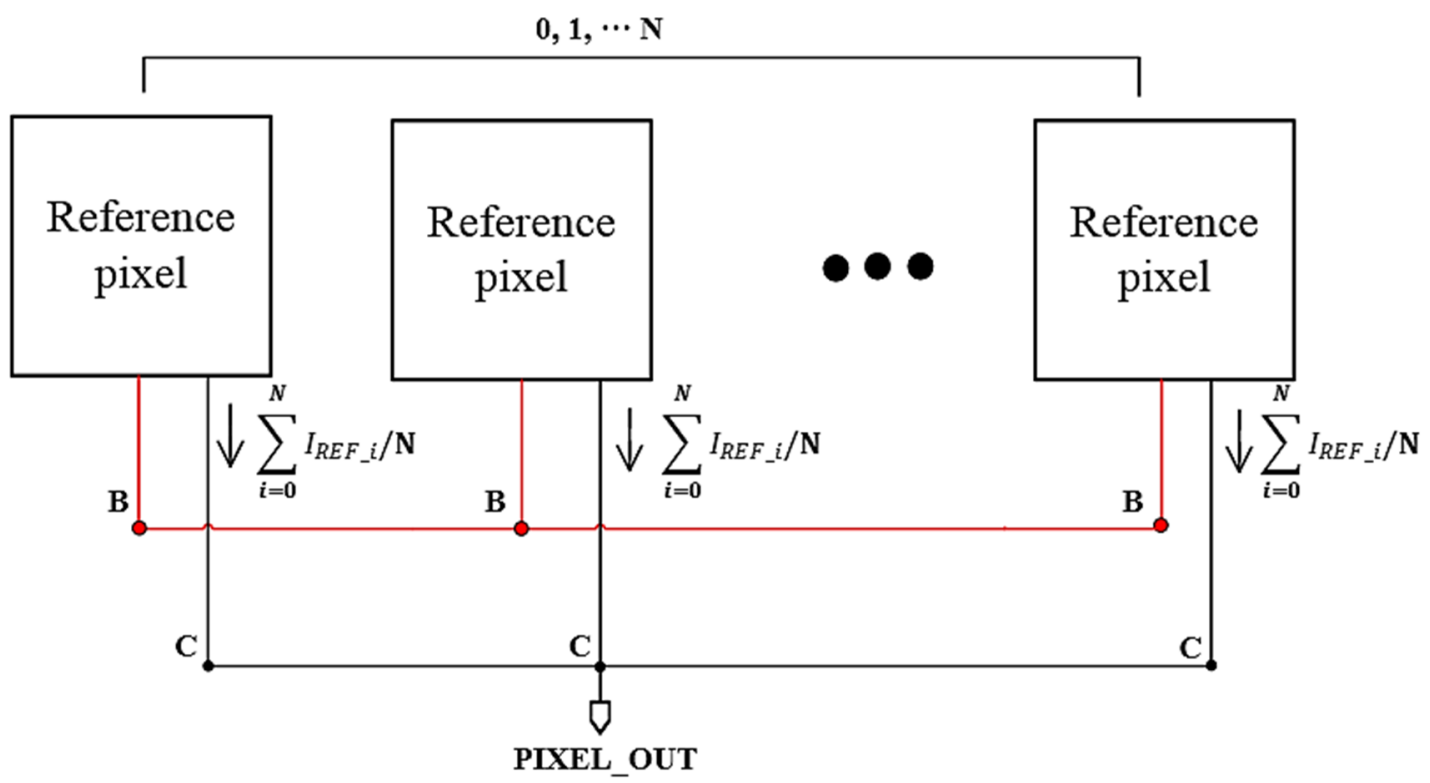

(a)

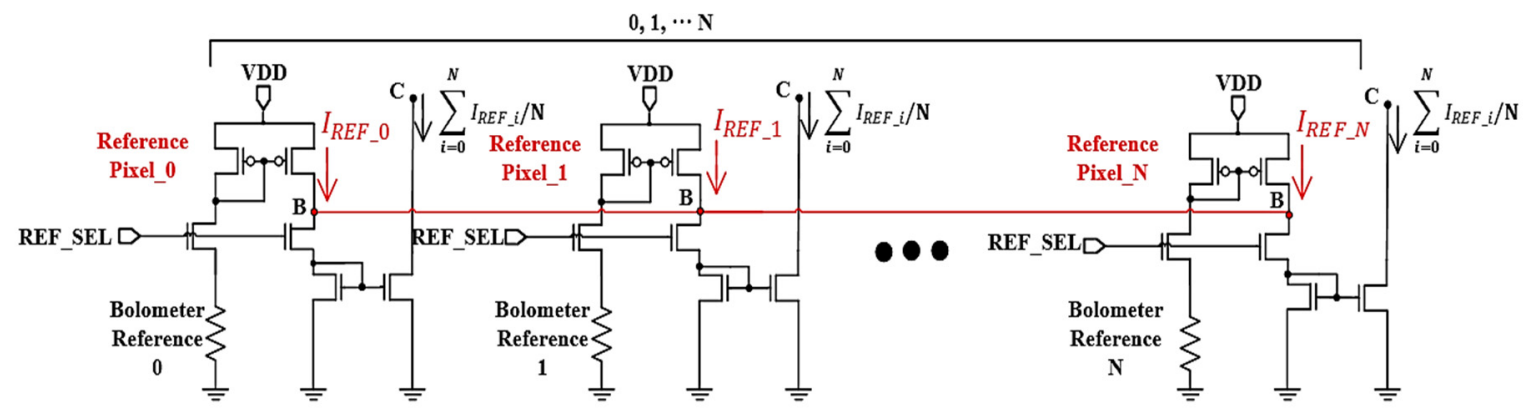

(b)

Figure 2. (a) Block diagram of averaging current generator using reference pixel, (b) schematic diagram of averaging current generator using reference pixel.

Figure 3 describes the resistance distribution of the active pixel. A bolometer with resistance deviations included for each active pixel was implemented with $90 \mathrm{k} \Omega, 100 \mathrm{k} \Omega$ and $110 \mathrm{k} \Omega$ resistors based on $100 \mathrm{k} \Omega$ resistances, taking into account $10 \%$ resistance deviation in the process. The array is composed of $4 \times 11$ active pixels. The bolometer included in the reference pixel consists of only $110 \mathrm{k} \Omega$ resistors, and it was fabricated using the standard CMOS process.

Figure 4a shows the layout of the designed readout circuit and Figure $4 \mathrm{~b}$ shows each column block diagram of the designed chip. The entire chip is based on column parallel structure. Each column of the entire chip is composed of buffer memory, line memory, a digital-analog converter (DAC), a calibration circuit, an active pixel, a reference pixel, a column readout circuit, and an ADC. The buffer memory, line memory, and DAC are used to control the calibration current $\left(I_{C A L}\right)$, which is a nano-ampere current. The column readout circuit converts current generated in a unit pixel into a voltage using an integrator based on a two-stage amp. 


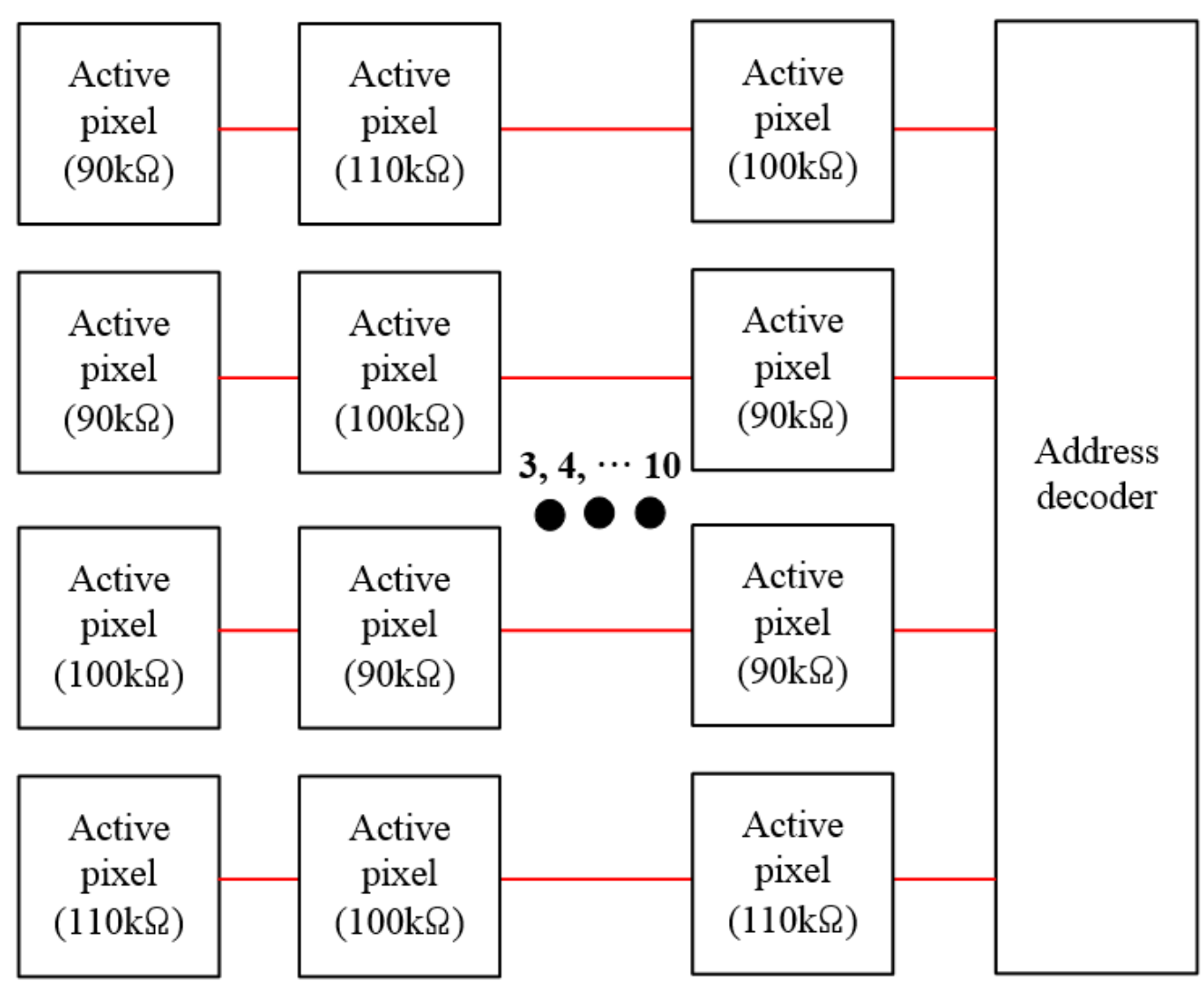

Figure 3. Resistance distribution of the active pixel.

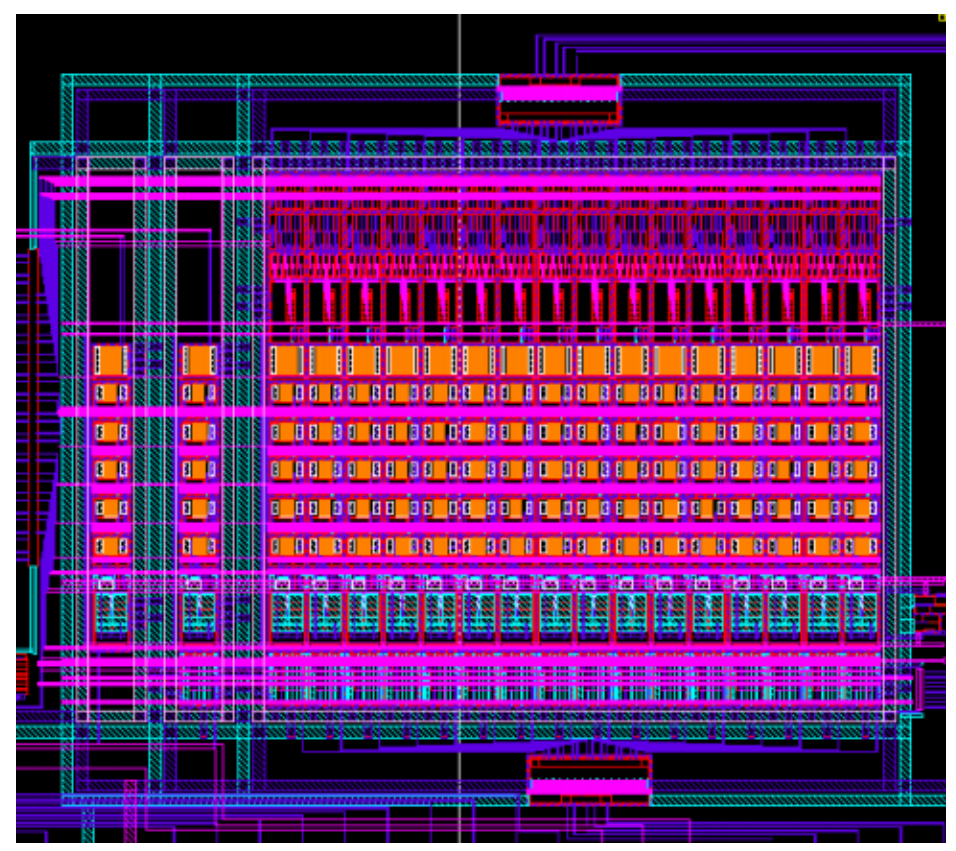

(a)

Figure 4. Cont. 


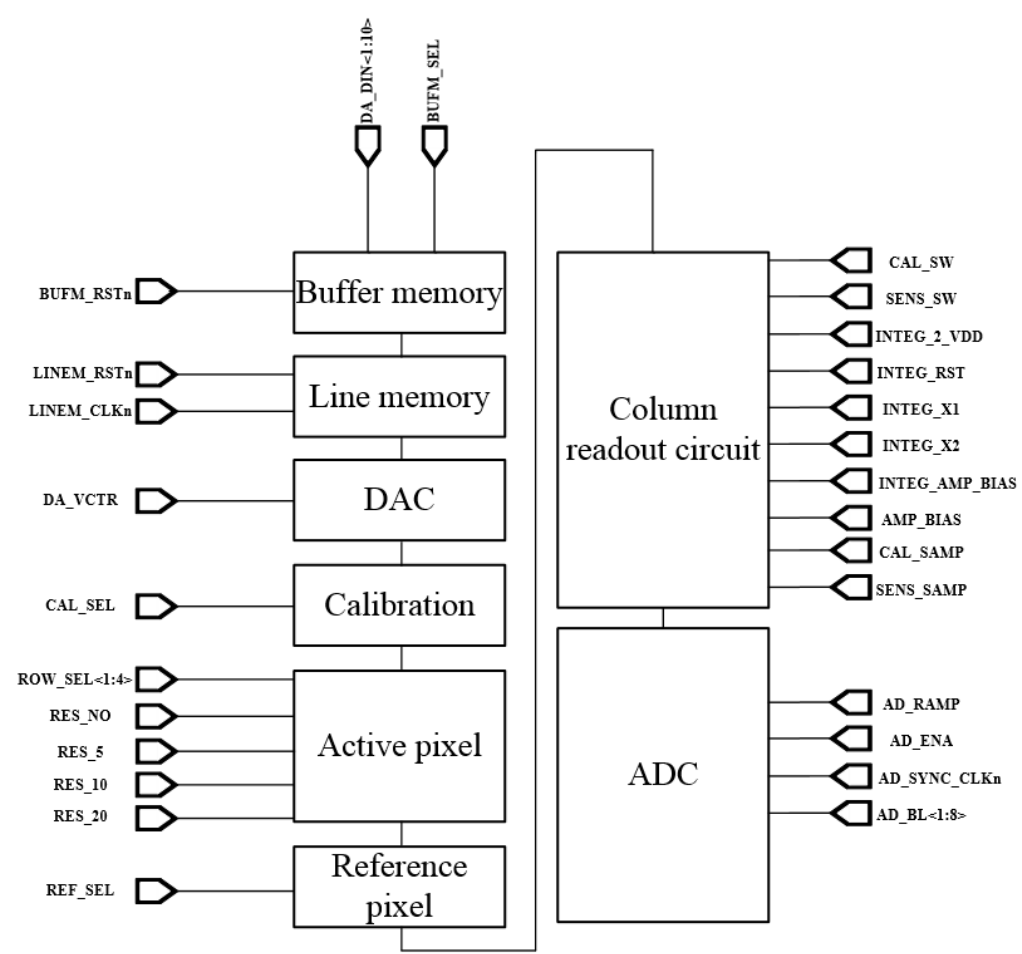

(b)

Figure 4. (a) Layout of designed readout circuit (b) each column block diagram of designed chip. DAC: digital analog converter; ADC: analog digital converter.

Figure 5a shows the schematic of the column readout circuit with integrator for current-to-voltage conversion, and Figure $5 b$ shows the timing diagram of the entire chip. The column readout circuit is composed of a current buffer for the current generated in the unit pixel, an integrator, a switch to control the gain of the integrator, a switch to reset the integrator, and a switch for sampling and selecting. The integrator is designed to control the output voltage gain based on a two-stage amplifier. The entire chip is based on a column parallel structure. Therefore, after the reset pulse (INTEG_RST) of the integrator is applied, the integrator output voltage is sequentially obtained for each row of the pixel. The integration time $\left(T_{i n t}\right)$ is the time from the reset of the integrator to the end of the operation of the sampling switch.

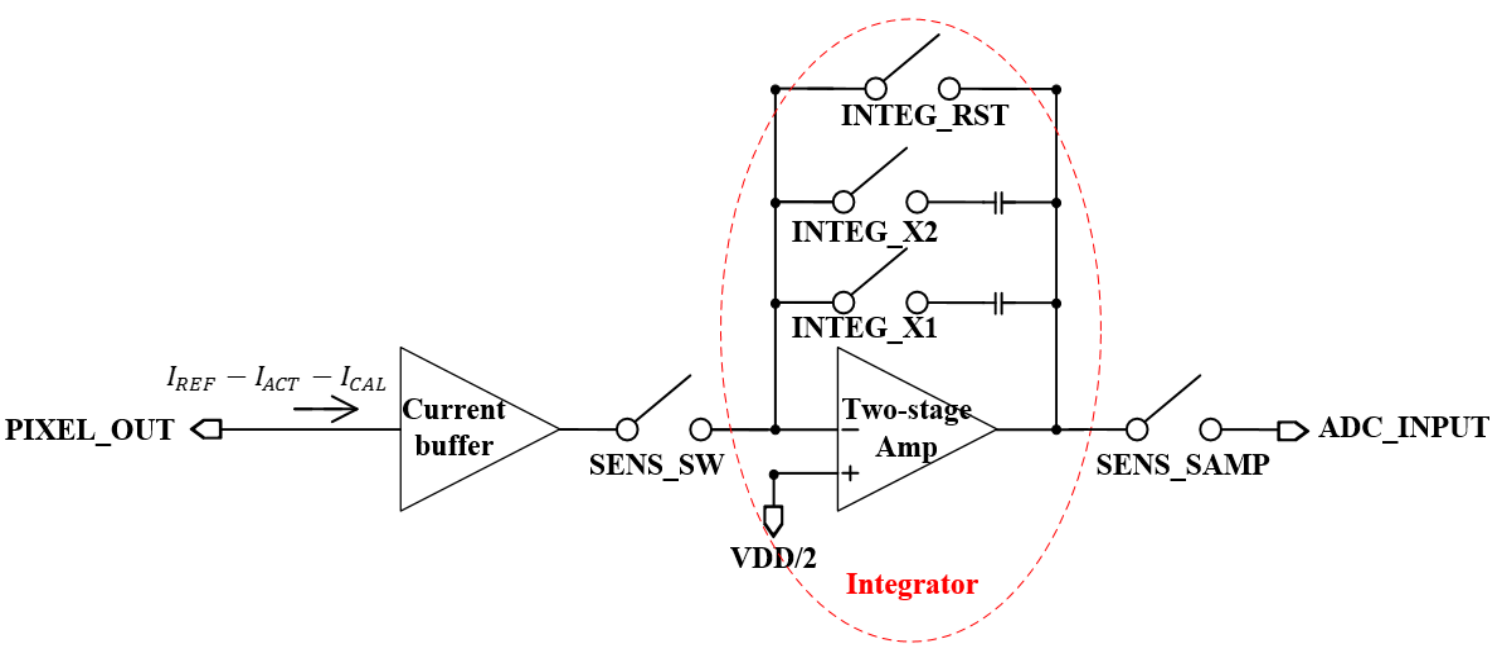

(a)

Figure 5. Cont. 


\begin{tabular}{|l|l|}
\hline BUF_EN & \\
\hline LMEM_EN & \\
\hline DAC_EN & \\
\hline CAL_EN & \\
\hline REF_EN & \\
\hline ROW_O & \\
\hline ROW_1 & \\
\hline ROW_2 & \\
\hline INTEG \\
RST
\end{tabular}

(b)

Figure 5. (a) Schematic of column readout circuit with integrator for current to voltage conversion, (b) timing diagram of entire chip.

\section{Measurement Results and Discussion}

In the case of the signal processing circuit of the bolometer-type uncooled infrared image sensor, it is important that the signal electrons generated by the infrared radiation or dark current of each pixel obtain the same output voltage for a certain integration time by the integrator. In order to verify the averaging pixel current adjustment technique, we compared the output current of the un-calibrated pixel and the integrator output voltage generated by the output current of the calibrated pixel according to the integration time.

Figure 6a shows the $90 \mathrm{k} \Omega$ active pixel output voltage according to the integrator reset voltage before calibration, and Figure $6 \mathrm{~b}$ shows the experimental results before and after applying the proposed averaging pixel current adjustment technique to the $90 \mathrm{k} \Omega$ active pixel. The entire pixel array is composed of $4 \times 11$ pixels. The active pixel integrated in the entire pixel array has a resistance of $90 \mathrm{k} \Omega$ and $110 \mathrm{k} \Omega$, which has a $10 \%$ process deviation, randomly integrated based on $100 \mathrm{k} \Omega$. Only a $110 \mathrm{k} \Omega$ resistor is integrated in the reference pixel. A $90 \mathrm{k} \Omega$ resistor has a $10 \%$ process deviation even at the same resistance value. After the reset of the integrator, the output voltage decreases to VDD/2 $(1.65 \mathrm{~V})$ according to the input current of the integrator generated by the resistor integrated in the active pixel. It was confirmed that the output voltage of the integrator before calibration was different for each active pixel due to the process deviation of the resistance. However, it was also confirmed that the output voltage of the integrator was nonlinear at the initial point after the reset of the integrator. Due to clock feedthrough by the integrator reset switch, the nonlinear output voltage is generated. Furthermore, the integration time is decreased as the deviation of the resistance of the active pixel and resistance of the reference pixel becomes larger. Therefore, it is assumed that the active pixel to which the adjustment technique is applied increases the integration time compared with the case where it is not. When the adjustment technique is applied in Figure $6 \mathrm{~b}$, it is confirmed that the uniform output voltage can be obtained even if the process deviation occurs at each $90 \mathrm{k} \Omega$ resistor in the active pixel. 


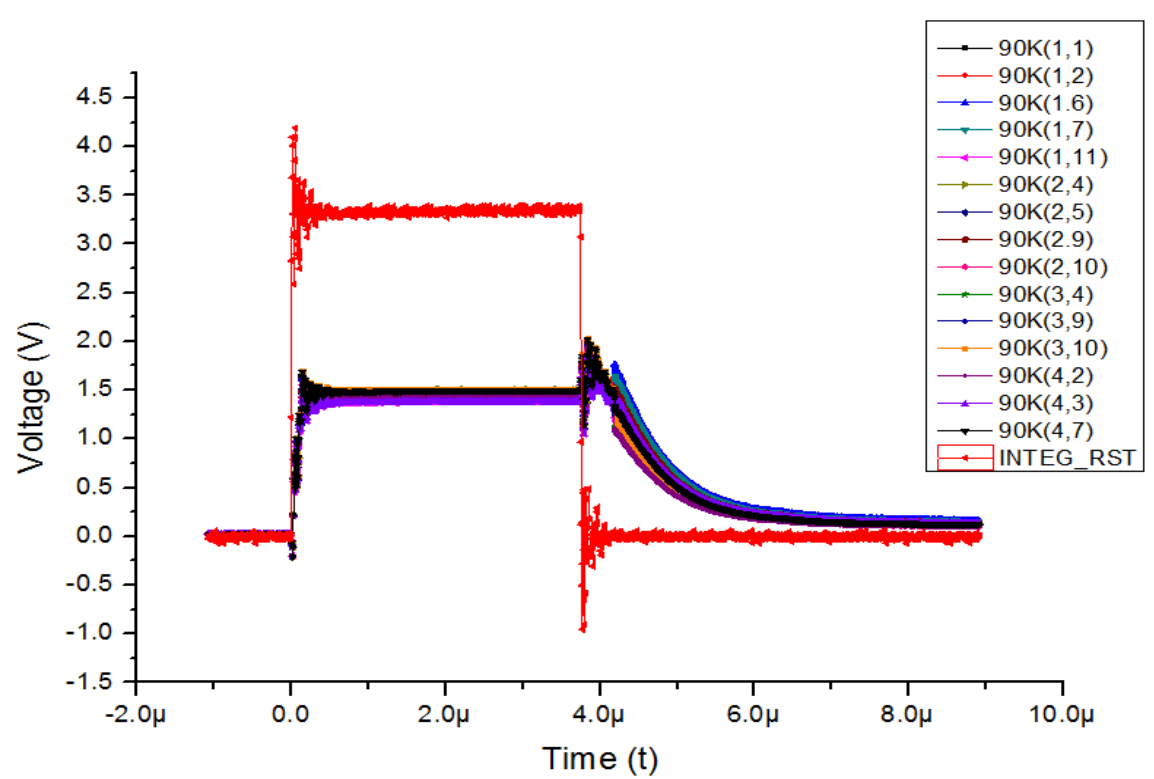

(a)

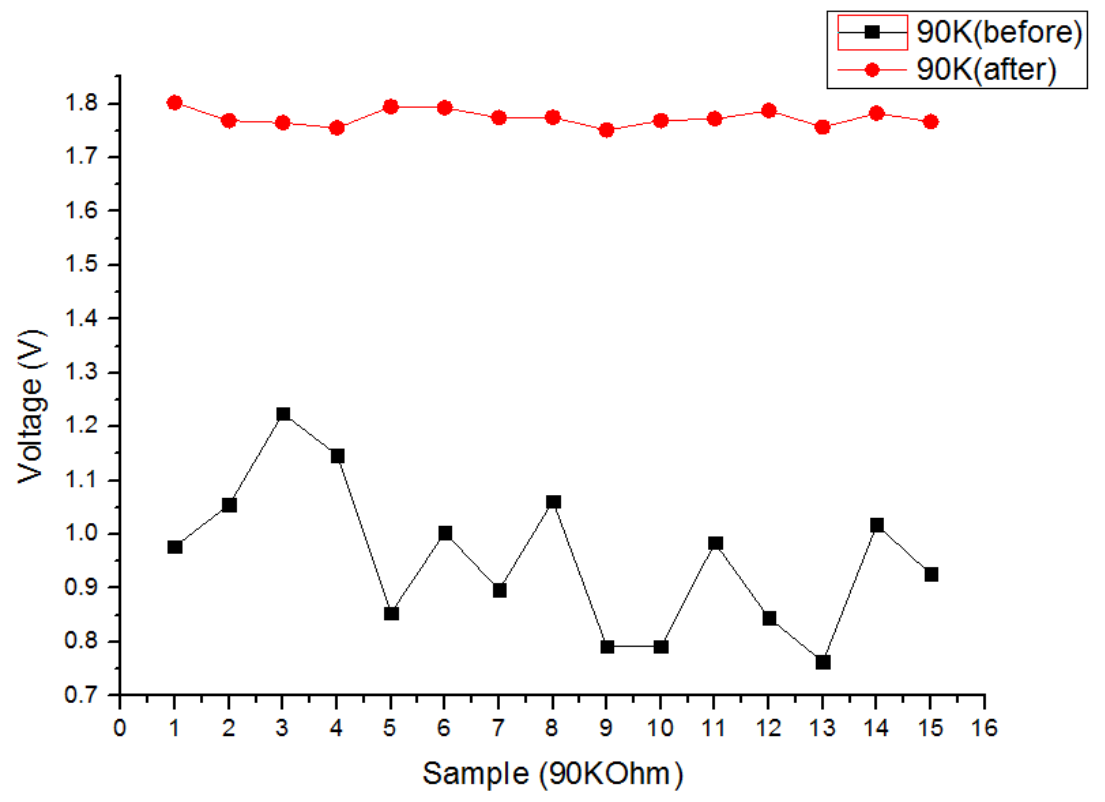

(b)

Figure 6. (a) The $90 \mathrm{k} \Omega$ active pixel output voltage according to integrator reset voltage before calibration (b) experimental results before and after applying proposed adjustment technique to the $90 \mathrm{k} \Omega$ active pixel.

Figure 7a shows the $100 \mathrm{k} \Omega$ active pixel output voltage according to the integrator reset voltage before calibration and Figure $7 \mathrm{~b}$ shows the experimental results before and after applying the proposed averaging pixel current adjustment technique of the $100 \mathrm{k} \Omega$ active pixel. Compared with the output voltage of the integrator when the resistance of the active pixel $90 \mathrm{k} \Omega$, the integration time is relatively increased. In addition a comparison with the output voltage of the integrator when the active pixel is $90 \mathrm{k} \Omega$, confirms that the error remains after calibration. Compared with the case in which the resistance of the active pixel is $90 \mathrm{k} \Omega$ and the resistance of the active pixel is $100 \mathrm{k} \Omega$, the resistance of the reference pixel is $110 \mathrm{k} \Omega$, so the $I_{R E F}-I_{A C T}$ current is relatively low. Therefore, not only the amount of current to be calibrated but also the absolute amount of deviation is reduced. A comparison of before and 
after the calibration, confirms that the red line increases to about $2 \mathrm{~V}$ in a Sample 11 of Figure $7 \mathrm{~b}$ because an instantaneous clock feedthrough occurs when the reset switch of the integrator is opened. The reason the difference between the voltage before and after the calibration is small in the case of Sample 9 of Figure $7 \mathrm{~b}$ is that the deviation of the active pixel resistance is about $+10 \%$, which is similar to the resistance of the reference pixel. However, the deviation of the integrator output voltage due to the resistance deviation based on $100 \mathrm{k} \Omega$ is shown in Figure 7a. In addition, it is confirmed that the resistance deviation among active pixels is also reduced by applying the averaging pixel current adjustment technique.

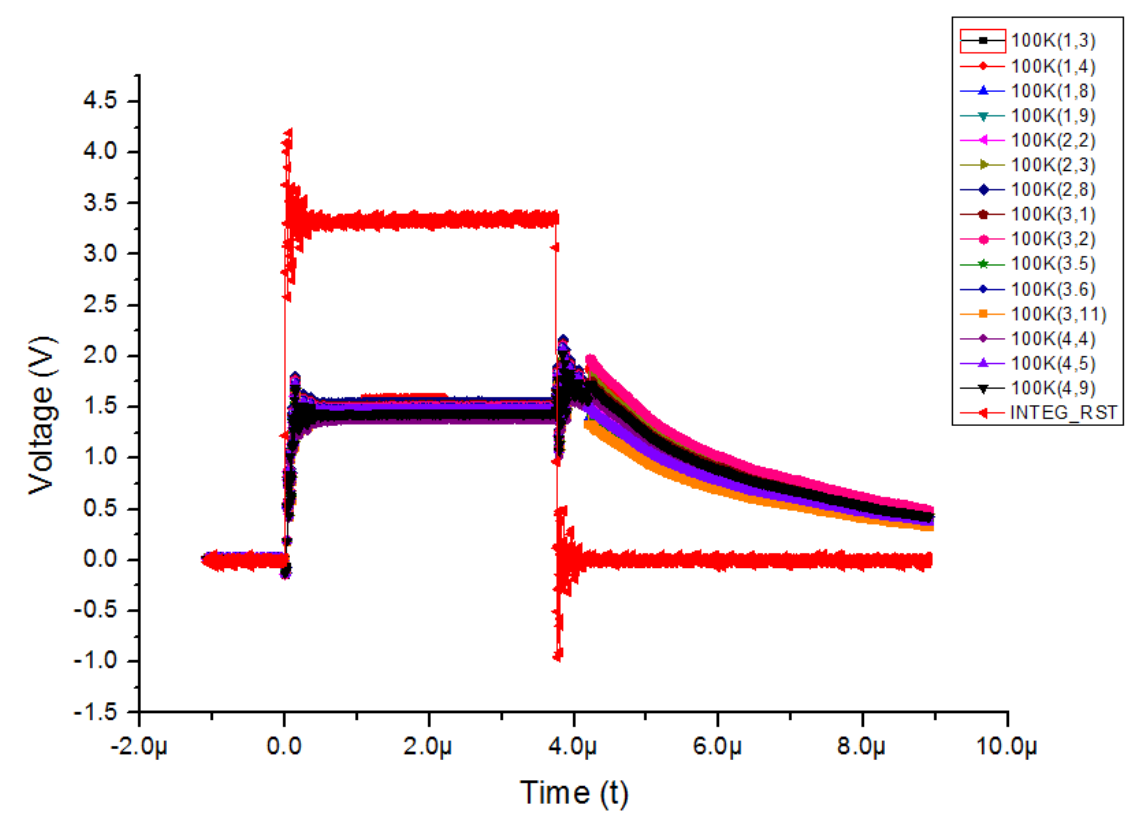

(a)

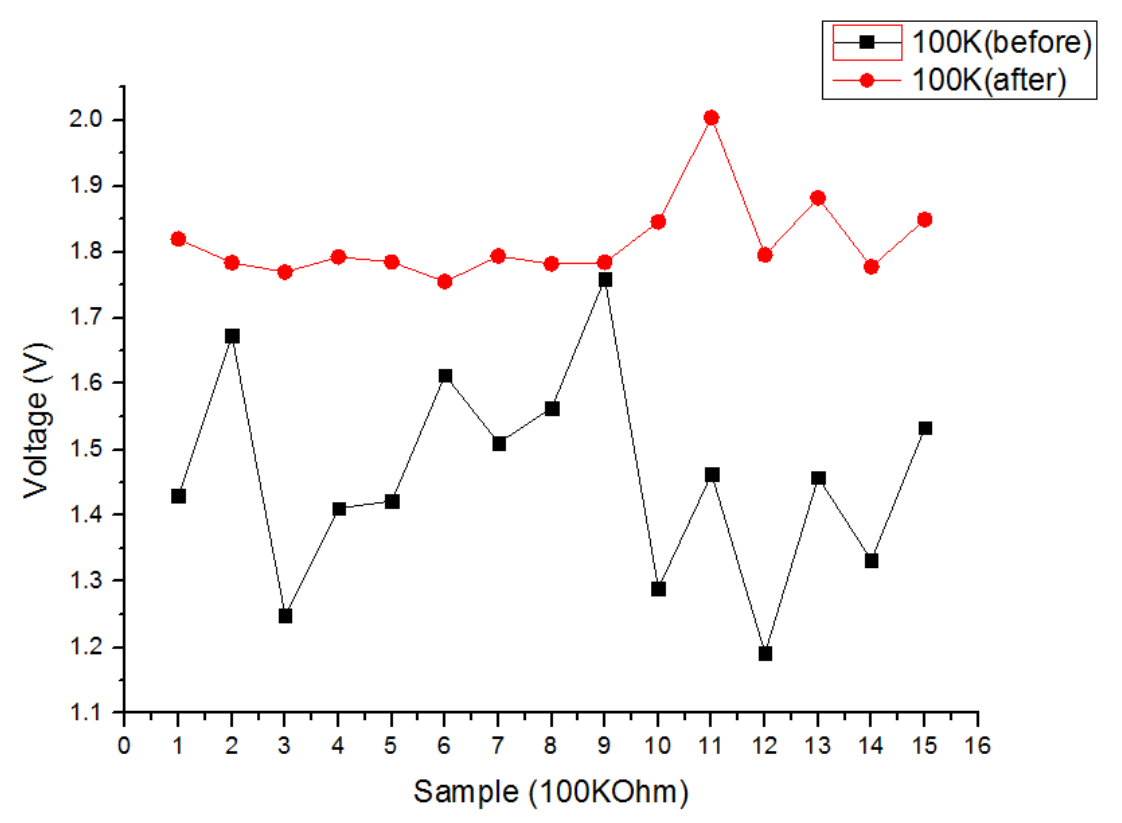

(b)

Figure 7. (a) The $100 \mathrm{k} \Omega$ active pixel output voltage according to integrator reset voltage before calibration (b) experimental results before and after applying proposed adjustment technique to the $100 \mathrm{k} \Omega$ active pixel. 
A 3D graph of before and after the proposed averaging pixel current adjustment technique of the entire array was applied is presented in Figure 8. In the $4 \times 11$ array where the resistance of the active pixel is integrated $90 \mathrm{k} \Omega, 100 \mathrm{k} \Omega$, and $110 \mathrm{k} \Omega$ randomly, the voltage which is on the Z-axis, is the output voltage of the integrator. Figure 8 a shows that before applying the averaging pixel current adjustment technique, the standard deviation of the integrator output voltage due to the output current among the active pixels is 0.385 . The standard deviation is the deviation amount of each active pixel when the output voltage of the active pixel is averaged. In addition, the standard deviation is FPN occurring between the active pixels due to the resistor's process deviation. Figure $8 \mathrm{~b}$ is a $3 \mathrm{D}$ graph showing after the averaging pixel current adjustment technique is applied. Compared with Figure 8a, the slope is relatively gradual and confirmed by measurement. The standard deviation between each active pixel after applying the averaging pixel current adjustment technique is 0.043 . Therefore, it is confirmed that the proposed averaging pixel current adjustment technique reduces the FPN by about $34 \%$.

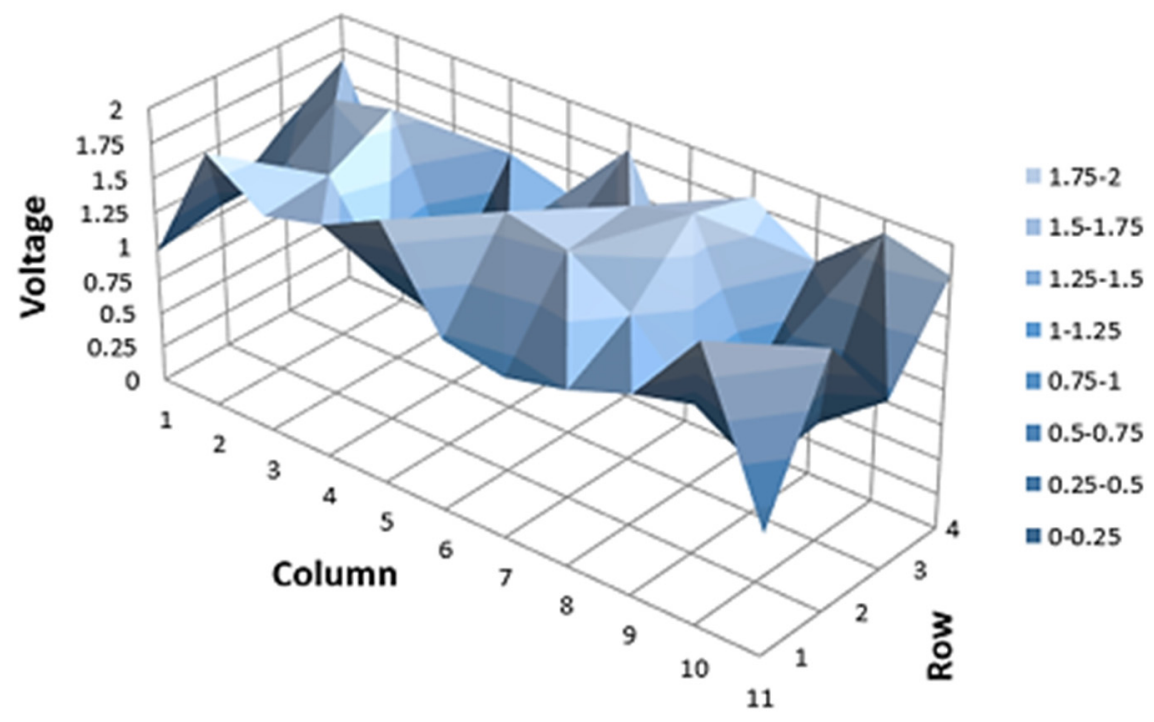

(a)

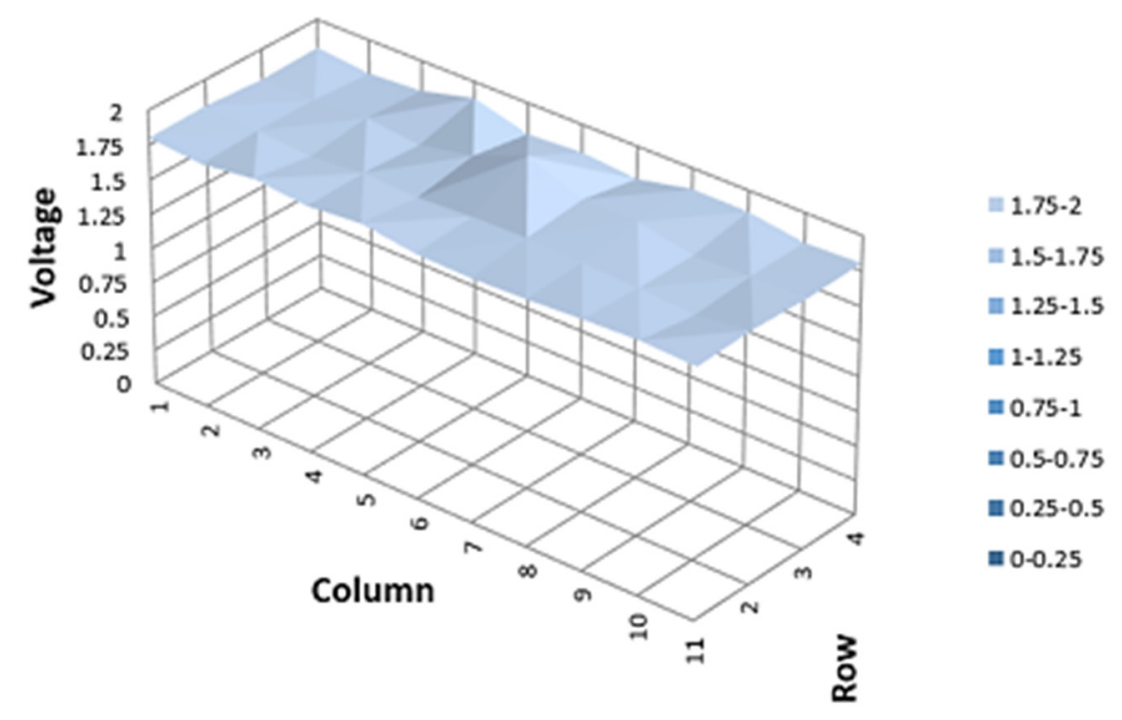

(b)

Figure 8. 3D graph of before (a) and after (b) applying proposed adjustment technique to the entire array. 


\section{Conclusions}

In this paper, we proposed an averaging pixel current adjustment technique for reducing FPN in the bolometer-type uncooled infrared image sensor. In order to reduce the resistance deviation causing the FPN among pixels of the bolometer-type uncooled infrared image sensor, an averaging pixel current adjustment technique was successfully implemented using a standard $0.35 \mu \mathrm{m}$ CMOS process. According to the experimental results, it was confirmed that the standard deviation of the output voltage according to the resistance of the active pixel was reduced by about $34 \%$ when the active pixel output voltages before and after the calibration were compared. Therefore, it was concluded that the output offset current according to the deviation of the bolometer resistance of each pixel was reduced by controlling the output code of the ADC using the proposed technique. We expect the proposed averaging pixel current adjustment technique to be effectively applied to the bolometer-type uncooled infrared image sensor for reducing FPN.

Author Contributions: This work was realized through the collaboration of all authors. S.-H.K., B.-S.C., J.L. (Jimin Lee), J.L. (Junwoo Lee), and J.L. (Jewon Lee) performed the simulations and experiments. J.-K.S., J.-H.P., and K.-I.L. analyzed the results and guided the research direction. S.-H.K. and J.-K.S. wrote the paper.

Funding: This research was supported by the Industrial Core Technology Development Program (Project No. 10052933) and Ministry of Trade, Industry \& Energy, MOTIE.

Conflicts of Interest: The authors declare no conflict of interest.

\section{References}

1. Kim, M.; Park, S.; Lee, K.; Yoo, H. Uncooled Infrared Micro-Bolometer FPA for Multiple Digital Correlated Double Sampling. IEEE Photonics Tec. Lett. 2018, 30, 517-520.

2. Parish, W.J.; Woolaway, J.T.; Kincaid, G.; Heath, J.L.; Frank, J.D. Low Cost $160 \times 128$ uncooled infrared sensor array. Proc. SPIE 1998, 3360, 111-119.

3. Deng, Y.-Z.; Tang, S.-F.; Zeng, H.-Y.; Wu, Z.-Y.; Tung, D.-K. Experiments on Temperature Changes of Microbolometer under Blackbody Radiation and Predictions Using Thermal Modeling by COMSOL Multiphysics Simulator. Sensors 2018, 18, 2593.

4. Liu, D.; Fang, Z.; Lu, W.; Chen, Z.; Zhang, Y. A Low-Power Low-Noise $160 \times 12017 \mu \mathrm{m}$ Pixel Pitch TEC-Less Uncooled Infrared Image Sensor. In Proceedings of the 2016 13th IEEE International Conference on Solid-State and Integrated Circuit Technology, Hangzhou, China, 25-28 October 2016; pp. 662-664.

5. Lv, J.; Que, L.; Wei, L.; Zhou, Y.; Liao, B.; Jiang, Y. Uncooled microbolometer infrared focal plane array without substrate temperature stabilization. IEEE Sens. J. 2014, 14, 1533-1544. [CrossRef]

6. Shen, N.; Yu, J.; Tang, Z. An uncooled infrared microbolometer array for low-cost applications. IEEE Photonics Technol. Lett. 2015, 27, 1247-1249. [CrossRef]

7. Dupont, B.; Dupret, A.; Becker, S.; Hamelin, A.; Guellec, F.; Imperinetti, P.; Rabaud, W. A $\left[10{ }^{\circ} \mathrm{C} ; 70{ }^{\circ} \mathrm{C}\right] 640$ $\times 48017 \mu \mathrm{m}$ Pixel Pitch TEC-Less IR Bolometer Imager with Below $50 \mathrm{mK}$ and Below 4 V Power Supply. In Proceedings of the 2013 IEEE International Solid-State Circuits Conference Digest of Technical Papers, San Francisco, CA, USA, 17-21 February 2013.

8. Hu, B.; Li, P.; Ruan, A.W.; Lin, D.M. Design and verification of $160 \times 120$ bolometer ROIC. In Proceedings of the 2011 International Conference on Computational Problem-Solving (ICCP), Chengdu, China, 21-23 October 2011; pp. 275-278.

9. Park, S.; Cho, T.; Kim, M.; Park, H.; Lee, K. A shutter-less micro-bolometer thermal imaging system using multiple digital correlated double sampling for mobile applications. In Proceedings of the 2017 Symposium on VLSI Circuits, Kyoto, Japan, 5-8 June 2017; pp. C154-C155.

10. Sizov, F.; Reva, V.; Golenkov, O.; Petriakov, V.; Shevchik-Shekera, A.; Korinets, S.; Sakhno, M.; Lysiuk, I.; Zabudsky, V.; Bunchuk, S.; et al. THz/sub-THz direct detector challenges: Rectification and thermal detectors for active imaging. In Proceedings of the 2014 20th International Conference on Microwaves, Radar and Wireless Communications (MIKON), Gdansk, Poland, 16-18 June 2014.

11. Roics, S.M.; Abbasi, S.; Shafique, A.; Ceylan, O.; Kaynak, C.B.; Kaynak, M. A Test Platform for the Noise Characterization of. IEEE Sens. J. 2018, 18, 6217-6223. 
12. Seo, I.W.; Jung, E.S.; Sung, M.Y. An Analog Front-End IC Design for $320 \times 240$ Microbolometer Array Applications. IEEE Trans. CIRCUITS Syst. 2015, 62, 1048-1052. [CrossRef]

13. Lv, J.; Zhong, H.; Zhou, Y.; Liao, B.; Wang, J.; Jiang, Y. Model-Based Low-Noise Readout Integrated Circuit Design for Uncooled Microbolometers. IEEE Sens. J. 2013, 13, 1207-1215. [CrossRef]

14. Jiang, Z.; Men, L.; Wan, C.; Xiao, P.; Jiang, C.; Tu, X. Low-Noise Readout Integrated Circuit for Terahertz Array Detector. IEEE Trans. Terahertz Sci. Technol. 2018, 8, 350-356. [CrossRef]

15. Seo, J.; Kim, G.; Lim, K.; Seok, C.; Kim, H.; Im, S.; Kim, J.; Kim, C.; Ko, H. An analog front-end IC with regulated R-I amplifier and CDS CTIA for microbolometer. In Proceedings of the 2013 13th International Conference on Control, Automation and Systems, Gwangju, Korea, 20-23 October 2013.

16. Ye, M.; Zhao, G.; Li, Y.; Zhao, Y. An Analog-Front ROIC with On-Chip Non-Uniformity Compensation for Diode-Based Infrared Image Sensors. Sensors 2019, 19, 298. [CrossRef] [PubMed]

17. Arnaboldi, C.; Pessina, G. A Front-End Readout for Micro Bolometers Having Sub Nano Volt Noise Floor. IEEE Trans. Nucl. Sci. 2006, 53, 2861-2868. [CrossRef]

18. Kim, K.-D.; Park, S.; Yoon, K.-S.; Kang, G.-G.; Han, H.-K.; Choi, J.-S.; Ko, M.-W.; Cho, J.; Lim, S.; Lee, H.-M.; et al. A 100mK-NETD 100ms-Startup-Time $80 \times 60$ Micro- Bolometer CMOS Thermal Imager Integrated with a $0.234 \mathrm{~mm}^{2} 1.89 \mu \mathrm{V}_{\mathrm{rms}}$ Noise 12b Biasing DAC. In Proceedings of the 2018 IEEE International Solid-State Circuits Conference, San Francisco, CA, USA, 11-15 February 2018.

(c) 2019 by the authors. Licensee MDPI, Basel, Switzerland. This article is an open access article distributed under the terms and conditions of the Creative Commons Attribution (CC BY) license (http://creativecommons.org/licenses/by/4.0/). 